\title{
Tuning the thickness of electrochemically grafted layers in large area molecular junctions
}

\author{
T. Fluteau, ${ }^{1}$ C. Bessis, ${ }^{1}$ C. Barraud, ${ }^{1, a)}$ M. L. Della Rocca, ${ }^{1}$ P. Martin, ${ }^{2}$ J.-C. \\ Lacroix, $^{2}$ and P. Lafarge ${ }^{1}$ \\ 1. Université Paris Diderot, Sorbonne Paris Cité, MPQ, UMR 7162, CNRS, 75205 Paris Cedex 13, \\ France \\ 2. Université Paris Diderot, Sorbonne Paris Cité, ITODYS, UMR 7086, CNRS, 15 rue J.-A. de Baïf, \\ 75205 Paris Cedex 13, France
}

\begin{abstract}
We have investigated the thickness, the surface roughness and the transport properties of oligo(1-(2bisthienyl)benzene) (BTB) thin films grafted on evaporated Au electrodes thanks to a diazoniumbased electro-reduction process. The thickness of the organic film is tuned by varying the number of electrochemical cycles during the growth process. Atomic force microscope (AFM) measurements reveal the variation of the thickness in the range of 2-27 nm. This variation displays a linear dependence with the number of cycles followed by a saturation attributed to the insulating behavior of the organic films. Both ultrathin $(2 \mathrm{~nm})$ and thin $(12$ and $27 \mathrm{~nm})$ large area BTB-based junctions have then been fabricated using standard CMOS processes and finally electrically characterized. The electronic responses are fully consistent with a tunneling barrier in case of ultrathin BTB film whereas for thicker molecular films a pronounced rectifying behavior is reported.
\end{abstract}

\section{INTRODUCTION}

Molecular electronics ${ }^{1}$ takes advantage of the huge variety of chemical functions offering a complete tunability of the electronic properties of the metal/molecule/metal junctions. Devices functions like rectifiers, ${ }^{2}$ switches, ${ }^{3}$ memories, ${ }^{4}$ spin filtering, ${ }^{5}$ interference ${ }^{6}$ can be achieved. From materials and processes points of view, organic materials can be inserted in devices following different approaches, namely the processes of thin film deposition. In the past few years, different techniques have been used following two main ways: ${ }^{7}$ physical and chemical. Focusing on the chemical sides, self-assembled monolayers (SAMs) in solution are widely used. ${ }^{8}$ Their transport properties are, however, mainly investigated at the nanometer scale using local probe techniques such as conducting AFM and scanning tunneling microscopy and thanks to nanoscale devices. ${ }^{9,10}$ Besides, SAMs are intrinsically limited to sub-10 nm thick films. An alternative approach to produce molecular layers is the electrochemical way using the electro-reduction of diazonium salts. ${ }^{11-13}$ This method results in a stable and dense ${ }^{14,15}$ molecular layer strongly attached on a conducting surface by covalent bonds. It is for instance used to reduce oxide layers and long-term passivation of 
surfaces in case of reacting materials. ${ }^{16}$ In the field of molecular junctions, McCreery et al. ${ }^{17-}$ ${ }^{19}$ have integrated this process to form thin organic films inserted between two conducting electrodes. As we will explain later, advantages of such technique are the robustness and the stability of the molecular layer against the device fabrication processes compared to SAMs. More importantly, it has been proposed recently that the molecules could play a major role in junctions only beyond the limit of quantum tunneling (around $4-5 \mathrm{~nm}$ ). ${ }^{20}$ With respect to the length of the molecules, controlling the thickness of the molecular layer is thus a key issue for developing new molecular devices and for understanding fundamental issues as the transport physics. ${ }^{11}$

In this article, we demonstrate the control of the thickness of diazonium-based organic thin films of (1-(2-bisthienyl) benzene) (BTB) attached on gold surfaces. We found the dependence of the molecular layer thickness on the number of voltammetry cycles with fixed potential boundaries. Our results demonstrate the ability to grow a homogeneous organic layers with a thickness ranging from 2 to $27 \mathrm{~nm}$. Current density-voltage (J-V) characteristics have been measured for 2, 12, and $27 \mathrm{~nm}$ thick BTB layers inserted between gold electrodes showing drastic change in their variation.

\section{THIN FILM ELABORATION: ELECTROCHEMISTRY}

A long $\mathrm{Au}$ electrode of $20 \mu \mathrm{m}$ width and $150 \mathrm{~mm}$ length is first patterned by optical lithography on a $\mathrm{SiO}_{2} / \mathrm{Si}$ substrate and by subsequent evaporation/lift-off steps. In this study, two different electrode thicknesses have been used: $20 \mathrm{~nm}(\mathrm{Ti}(5 \mathrm{~nm}) / \mathrm{Au}(15 \mathrm{~nm}))$ and $32 \mathrm{~nm}$ (Ti $(2 \mathrm{~nm}) / \mathrm{Au}(30 \mathrm{~nm}))$. The Au electrode is connected by design to a wide sacrificial pad which allows electrical contacts with an alligator clip to impose an electrochemical potential over the metallic region.

\footnotetext{
a) Author to whom correspondence should be addressed. Electronic mail: clement.barraud@univ-paris-diderot.fr
} 
The electro-grafting of the organic layer is then performed using cyclic voltammetry. The Au electrode described above is used as a working electrode and immersed in an organic solution containing the amino-derivative of bisthiophene benzene (1-(2-bisthienyl)-4-aminobenzene (BTB)) as shown in the inset of Fig. 1(a). By adding an amount of tertbutyl nityrite (18 $\mu$, 30 eq.), the diazonium salt is fabricated in-situ and the cyclic voltammetry technique allows its electro-reduction and generates the aryl radical. ${ }^{21}$ Fig. 1(a) shows the evolution of the cyclic voltammogram of the electrode in the grafting solution up to the $50^{\text {th }}$ cycle. The first cycle shows the characteristic reduction peak close to $0 \mathrm{~V} / \mathrm{SCE}$ (Saturated Calomel Electrode) of the diazonium group. ${ }^{14}$ The second and following cycles reveal a drastic decrease of the cathodic current demonstrating that a blocking behavior of the Au electrode is achieved by the deposited molecules during the first cycle. This observation is in a perfect agreement with a previous study ${ }^{22}$ reporting the passivation of an electrode by an ultrathin film described as an oligo-BTB layer.
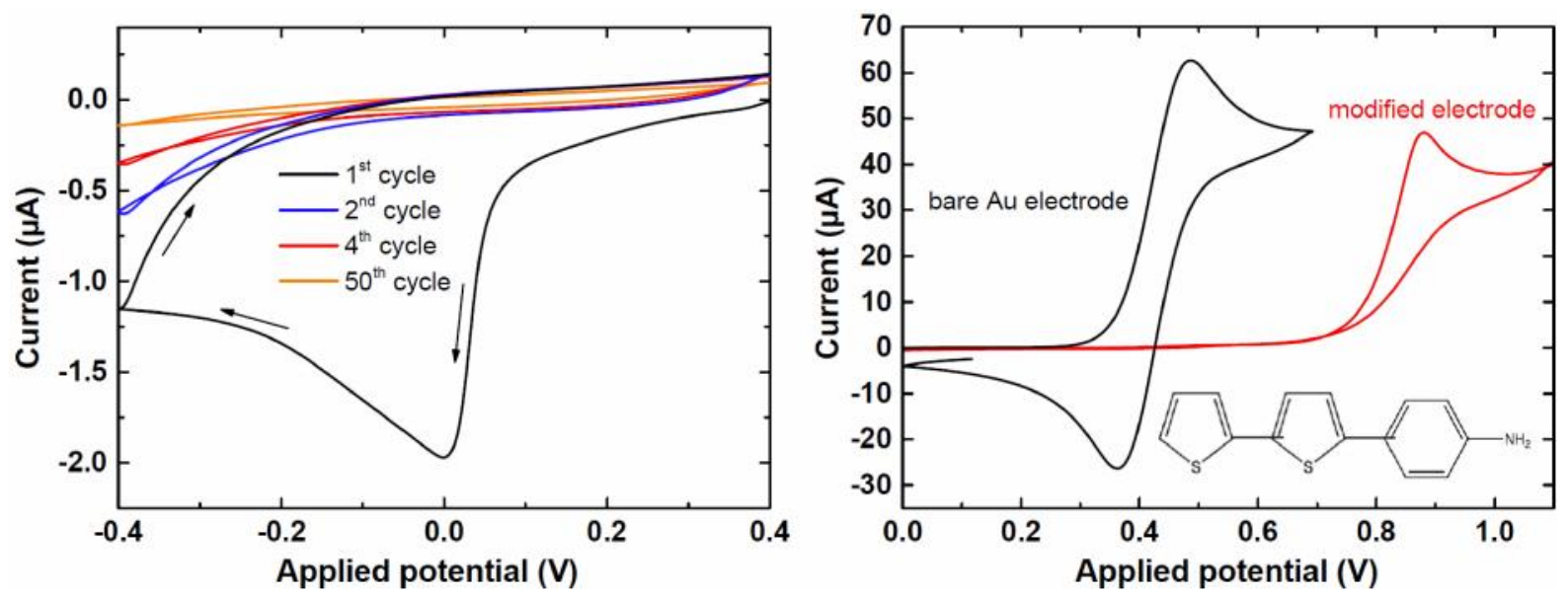

FIG. 1. (a) Cyclic voltammograms of a patterned gold electrode in a solution of $0.1 \mathrm{M}$ $\mathrm{NBu}_{4} \mathrm{BF}_{4}+5 \mathrm{mM}$ BTB in acetonitrile. The scan rate is $0.1 \mathrm{~V} / \mathrm{s}$. (b) Cyclic voltammograms of the bare (black line) and modified (red line) electrodes in an organic solution of ferrocene $5 \mathrm{mM}+0.1 \mathrm{M}$ $\mathrm{NBu}_{4} \mathrm{BF}_{4}$ (scan rate $=0.1 \mathrm{~V} / \mathrm{s}$ ). The BTB layer is electro-active and becomes p-doped at a potential close to $0.5 \mathrm{~V} / \mathrm{SCE}{ }^{22}$ Inset shows a BTB monomer.

To prove the presence and the homogeneity of the organic layer, we investigate on Fig. 1(b) the electro-activity of ferrocene $(\mathrm{Fc})$ on a bare and an oligo-BTB modified electrodes. In the potential window $(0 \mathrm{~V} / \mathrm{SCE}<\mathrm{E}<0.6 \mathrm{~V} / \mathrm{SCE})$ where Fc redox reactions are seen on a bare 
electrode (black curve in Fig. 1(b)), no current is observed (red curve in Fig. 1(b)) on the oligo-BTB/Au electrode. Indeed the BTB layer blocks the electron transfer from the metallic surface toward Fc demonstrating the homogeneity of the film over the entire surface. The presence of pinholes would be revealed by a slight increase of current around the standard potential of the redox probes $(0.4 \mathrm{~V} / \mathrm{SCE})$. For scanning beyond $0.5 \mathrm{~V} / \mathrm{SCE}$ the Fc oxidation peak finally appears, the BTB layer is electro-active and becomes p-doped at this particular

potential value. ${ }^{22-24}$ We now focus on the atomic force microscopy (AFM) characterizations of this molecular film.

\section{AFM STUDY OF THE GRAFTED ORGANIC FILM}

After the electro-grafting process, the samples are rinsed in pure ethanol and dried with a nitrogen flow. An AFM with ultrasharp tip (radius of $\sim 10 \mathrm{~nm}$ ) is used to characterize the thickness and the topography of the BTB film with respect to the number of grafting cycles. It is set in the tapping mode to avoid any deterioration of the soft molecular layer while scanning.

First of all, we checked the surface roughness of the bare Au electrodes for the different thicknesses of 20 and $32 \mathrm{~nm}$ as determined by the quartz balance during the growth made by evaporation at a rate of $0.5 \mathrm{~nm} / \mathrm{s}$. Highly resolved scans are performed over the metallic surface. Typical RMS roughnesses of the order of $0.4 \mathrm{~nm}$ on a $7 \times 7 \mathrm{~m}^{2}$ area are found meaning that the surface is relatively smooth and flat and thus suitable for devices.

On grafted samples, the BTB thickness is then determined by substracting the thickness of the $\mathrm{Au}$ bottom electrode from that of the Au/BTB modified electrode measured by AFM following a statistical approach. In order to minimize the total thickness and to warranty qualitative step height measurements, the $32 \mathrm{~nm}$ thick electrodes are used for the samples from 1 to 9 grafting cycles (ultrathin organic film). The $20 \mathrm{~nm}$ thick electrode are used for the 
thicker organic film with 10 and above grafting cycles. Inset of Fig. 2(a) shows a typical AFM image of an Au/BTB step over an area of $5 \times 5 \mu \mathrm{m}^{2}$. We realize identical topography scans on different Au/BTB (X cycles) samples in order to determine the evolution of the total thickness as a function of the number (X) of electro-grafting cycles. In Fig. 2(b), each point is an average value over ten different profiles. Error bars were determined thanks to statistics and their values is two times the standard deviation which correspond to a confidence interval of $95 \%$.

An important observation is that we can control the thickness of the molecular layer between 0 and $27 \mathrm{~nm}$. Then two different regimes during the molecular layer growth can be clearly distinguished: i) up to 10 cycles, the increase of the thickness is quite linear with an average slope of $2.1 \mathrm{~nm}$ per cycle, ii) above 20 cycles, the thickness reaches a saturation value of around $27 \mathrm{~nm}$. It seems that in this regime and within this scan window, the electrode surface is completely passivated and no more charge transfer is allowed. A similar saturation effect was found in the case of spontaneous grafting of aryldiazonium salts on carbon surface. ${ }^{25}$ The growth mechanism and the formation of multilayers are still under debate but it involves intermolecular binding between two radicals. The radicals are generated at the outer surface and can afterward react with the already grafted groups to form oligomers. ${ }^{26}$ It was shown that the apparent rate constant of this reaction decreases slowly with the layer thickness. After a dozen of nanometers the reaction was blocked by the grafted layers. ${ }^{25}$ 

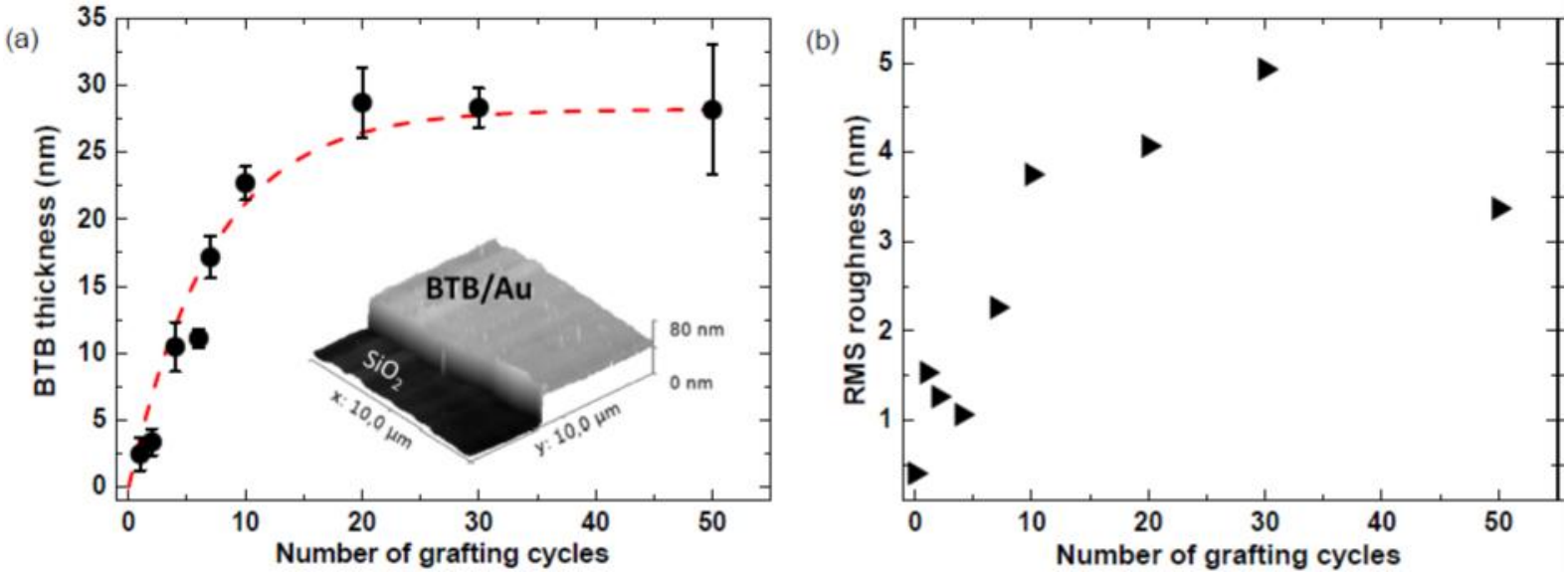

FIG. 2. (a) Thickness of the electro-grafted BTB film as a function of number of electro-chemical cycles. The dashed line is a guide for the eyes. Inset: three-dimensional AFM image (tapping mode) of a step between the $\mathrm{Si} / \mathrm{SiO}_{2}$ substrate and an electro-grafted BTB film (4 cycles)/Au bilayer. (b) The corresponding surface roughness of the film obtained over a $5 \times 5 \mu \mathrm{m}^{2}$ area is also given.

Surface RMS roughnesses are also extracted for the measurements by realizing highly resolved scans over the Au/BTB (X cycles) surface. Interestingly, one can remark also an increase of the roughness with respect to the number of cycles occurring abruptly above $\sim 10$ cycles, showing that once the molecular layer thickness starts to saturate, roughness increases. This could also indicate a change in the film morphology as one can remark on Fig.3 (a) and (b).
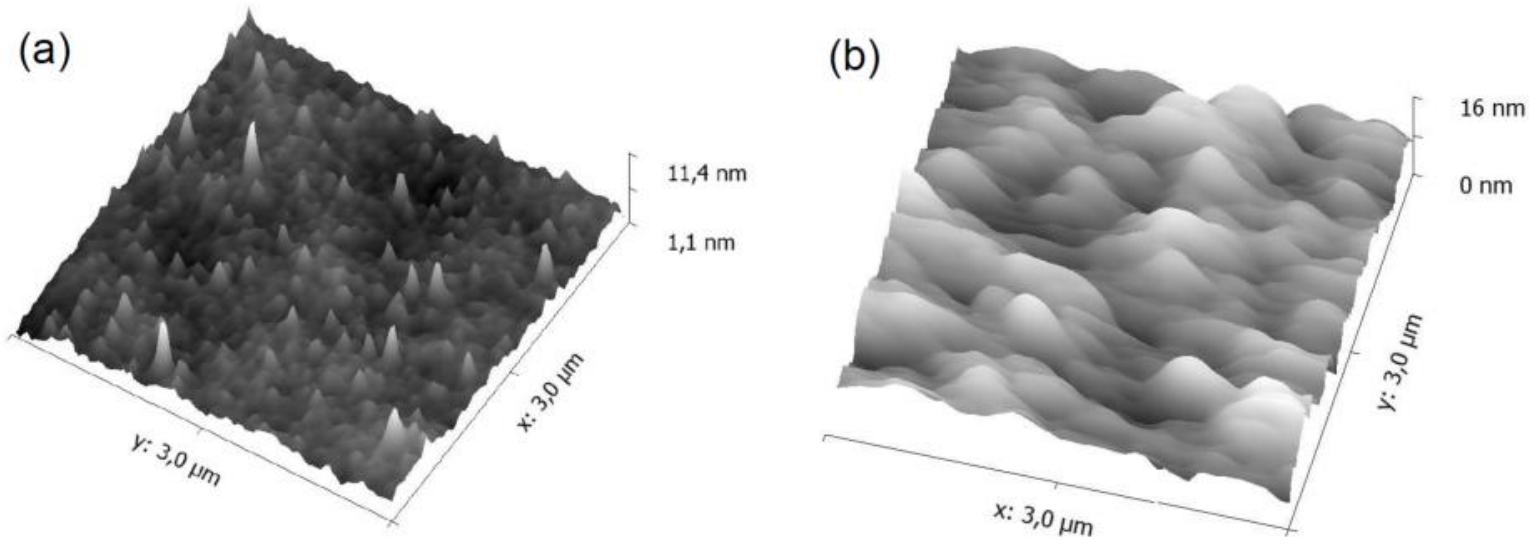

FIG. 3. (a) AFM image (tapping mode) of a grafted BTB layer on gold (2 cycles). (b) AFM image (tapping mode) of a grafted BTB layer on gold (30 cycles). 
Vertical molecular junctions are finally realized by contacting the Au/BTB (X cycle(s)) layers with an $\mathrm{Ti} / \mathrm{Au}$ top electrode in a cross-bar geometry thanks to an additional photolithography/lift-off step. Very low evaporation rate $(0.02 \mathrm{~nm} / \mathrm{s})$ are used during the metal evaporation $(\mathrm{Ti}(1 \mathrm{~nm}) / \mathrm{Au}(50 \mathrm{~nm}))$ to limit the diffusion of metal atoms through the organic thin film. Vacuum is also lowered to $10^{-8}$ mbar to avoid contamination. At the end of the fabrication process, vertical Au/BTB (X cycles)/Ti/Au junctions are obtained with a size of $20 \times 20 \mu \mathrm{m}^{2}$ as shown on Fig. 4(a).

Three different thicknesses of the organic film are studied here which are expected to lead to striking different transport regimes: a $2 \mathrm{~nm}, 12 \mathrm{~nm}$ and $27 \mathrm{~nm}$ thick films. Representative current density-voltage (J-V) characteristics obtained for the three junctions are shown in Fig. 4 (b) to (d). The yield, defined as the percentage of devices that showed neither short contacts nor resistive behavior, is around $70 \%$ for devices integrating a "thick" BTB layer and $40 \%$ for the $2 \mathrm{~nm}$ junctions. 
(a)

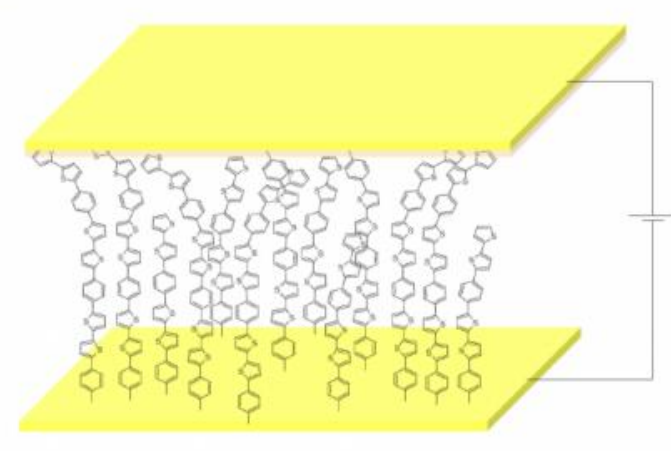

(c)

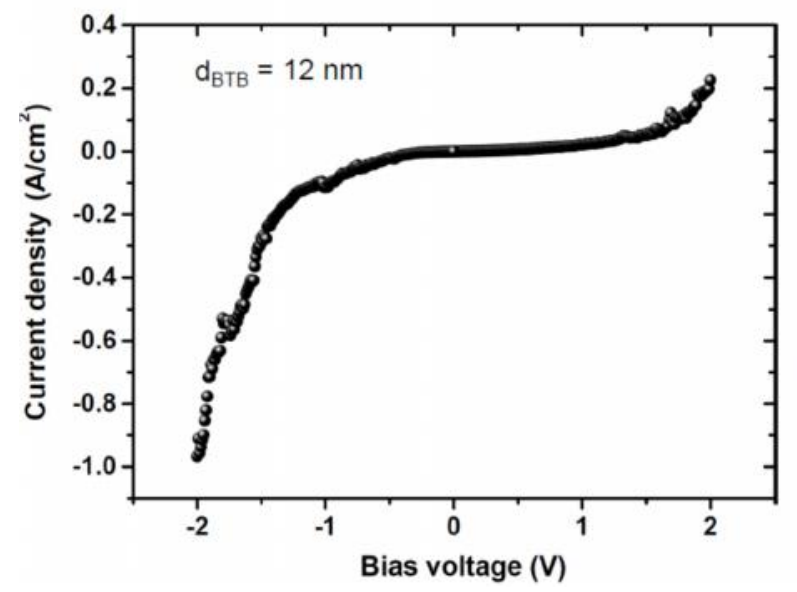

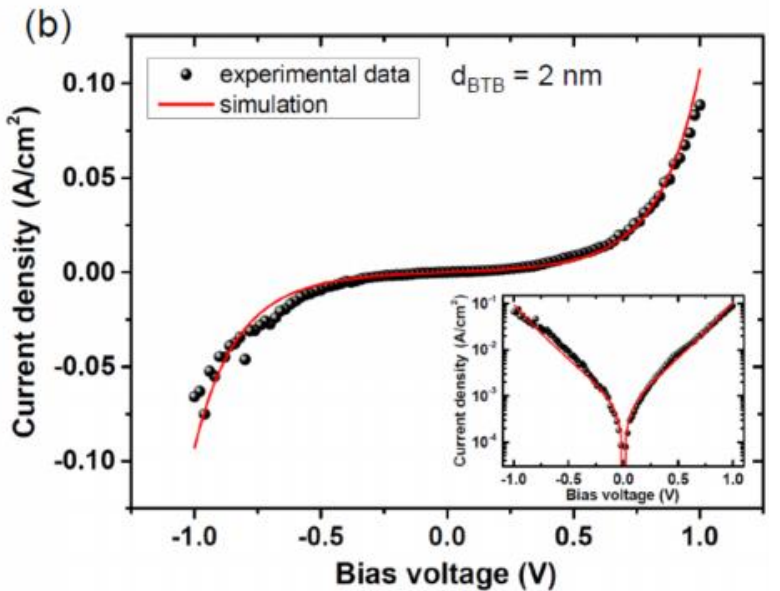

(d)

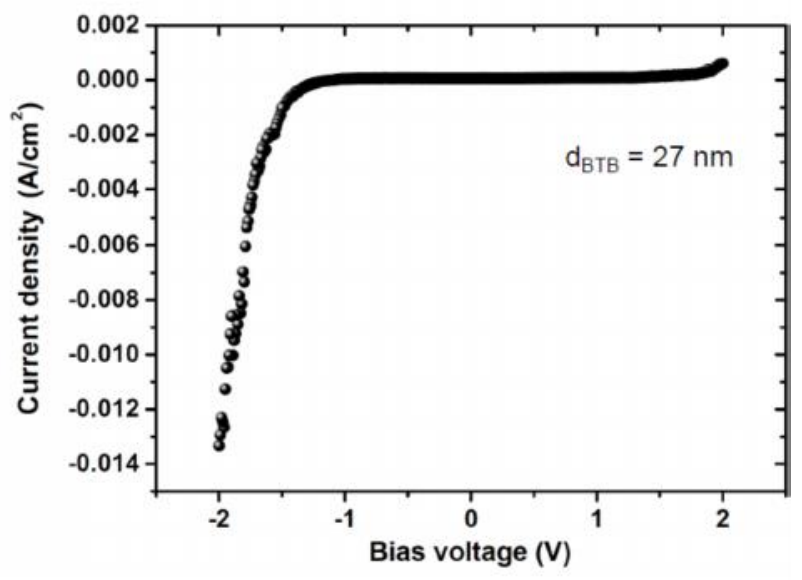

FIG. 4. (a) Scheme of a molecular junctions (Au/BTB/Ti/Au). (b) Typical tunneling curve obtained at $300 \mathrm{~K}$ in an Au/BTB (2nm)/Ti/Au molecular junction. A fit with a trapezoidal barrier model is also shown with the red continuous curve giving a tunneling barrier thickness of $2.2 \mathrm{~nm}$. Inset: same characteristic on a semi-log scale. (c) J-V characteristic obtained in the intermediate regime of thickness $(12 \mathrm{~nm})$. An asymmetry is present. Also note that the maximum applied bias voltage is now $2 \mathrm{~V}$. (d) $\mathrm{J}-\mathrm{V}$ characteristic obtained in an Au/BTB $(27 \mathrm{~nm}) / \mathrm{Ti} / \mathrm{Au}$ junction showing a complete rectifying behavior.

A J-V characteristic observed in a Au/BTB (2nm)/Ti/Au device (Fig. 4(b)) shows a nonmonotonic behavior and is rather symmetric comparing the negative and positive voltage polarizations. A low-bias resistance in the order of the $\mathrm{M}$ range can be extracted. $\mathrm{J}-\mathrm{V}$ characteristics with thicker BTB films (12 nm and $27 \mathrm{~nm}$ shown in Fig. 4 (c) and (d) respectively) are very different: even if showing a non-monotonic behavior, they are clearly asymmetric and they present a rectifying behavior with large current at negative polarity of the bias voltage. The average rectification ratio is 4 at $2 \mathrm{~V}$ for the $12 \mathrm{~nm}$ films and seems even to be more important by increasing the thickness of the organic film (18 at $2 \mathrm{~V}$ ). A diode 
behavior with current density of the order of few $10^{-1} \mathrm{~A} / \mathrm{cm}^{2}$ in the "ON" state was already reported by our group ${ }^{22}$ in junctions made with a $7 \mathrm{~nm}$ thick BTB film. Similar current densities were also reported in BTB junctions with carbon electrodes. ${ }^{11}$

This is a quite interesting result. Our fabrication procedure can result in large area, good quality, functional and robust junctions with an ultrathin organic film even thinner to what was reported earlier with BTB. ${ }^{11}$ It is worth to note that the stability of molecular junctions was also noticed down to the single diazonium-based molecule scale. ${ }^{27}$

Now, we discuss about the possible transport mechanisms occurring through the BTB film. In the case of an ultrathin film of $2 \mathrm{~nm}$ for low bias voltages $(<1 \mathrm{~V})$, BTB molecules have an insulating behaviour, ${ }^{11}$ tunneling transport between the two electrodes must be the dominant transport mechanism. It also explains the high value of the measured resistance also reported for polythiophene wires of similar lenghts ${ }^{28}$. A fit to the experimental data (continuous red line in Fig.3(a)) using a trapezoidal barrier model ${ }^{22}$ and which equations are given in the following was performed in order to evaluate the important transport parameters :

$$
J=\frac{4 \pi}{h^{3}} \int_{0}^{+\infty}\left[\begin{array}{lll}
f_{t} & (E)-f_{b} & (E)] d \int_{0}^{E} T\left(E_{\chi}\right) d E_{\chi}
\end{array}\right.
$$

With the transmission $T\left(E_{\chi}\right)=e . \quad\left\{-\frac{4 \pi}{n} \int_{S_{1}}^{s_{2}} \sqrt{\left[2 m \phi(x, V)-E_{\chi}\right]} d\right\}$ where the potential profile is expressed as follows: $\phi(x, V)=\phi_{t}+\frac{x}{d}\left(\phi_{b} \quad-e-\phi_{t_{i}}\right) . f_{\text {top }}$ and $f_{\text {bottom }}$ are the Fermi functions of the two electrodes, $s_{1}$ and $s_{2}$ are the classical turning point at $E_{x}, m$ is taken as the free electron mass, $V$ is the applied bias voltage and $d$ is the barrier thickness. It results in a potential barrier thickness of $2.2 \mathrm{~nm}$ (which roughly correspond to a dimer of BTB in series) in perfect agreement with the thickness estimated from AFM measurements. Barrier heights of $\phi_{\text {bottom }}=1.33 \mathrm{eV}$ and $\phi_{\text {top }}=1.47 \mathrm{eV}$ are also revealing a slight asymmetry of the junction. The fitting procedure assumes however a trapezoidal barrier and does not take into account the value of the effective mass which is unknown for BTB molecules, effect of 
the eventual presence of pinholes ${ }^{29,30}$ related to the large size $\left(20 \times 20 \mu \mathrm{m}^{2}\right)$ of the junctions and effect related to image forces that could be strong in molecular junction. ${ }^{31}$ In the case of a thicker film, direct tunneling between the two electrodes is not physically possible. In this case, intermediate states have to be invoked ${ }^{32}$ during the transport process across the molecules.

The origin of the observed asymmetry and the rectifying behavior are still under investigation. However two different possibilities can be put forward. First, the asymmetry could come from different electronic structures of the two interfaces as revealed by our tunneling experiment. It would highlight the different natures ${ }^{33,34}$ of the two metal/molecule interfaces. At the bottom interface, the BTB molecule is covalently attached to the gold electrode by a $\mathrm{C}$-Au chemical bond ${ }^{14}$ whereas at the top interface a thin adhesion layer of $\mathrm{Ti}$ is first evaporated followed by a layer of Au. Those two metals have different work functions. The presence of chemical bond between the top electrode and the Ti/Au layers is not expected but can not be completely excluded. ${ }^{33,35}$ Spectroscopy techniques must be employed to explore this possibility. ${ }^{36}$ The second explanations concerns the property of the organic layer itself which is supposed to behave like a diode. It should thus be more reflected in transport experiments in which charge transport occurs via at least one molecular orbital (not in the tunneling experiment). It is known that the electrochemical behavior of the molecule is expected to be highly asymmetric with respect to the electrochemical potential. ${ }^{24}$ When the bottom electrode is negatively polarized (negative bias in our measurement configuration), the organic layer is supposed to become p-doped $\left(\mathrm{BTB}^{+}\right)$and highly conducting compared to the neutral state. ${ }^{24}$ This doping effect is not symmetric with respect to the bias voltage. It is interesting to note that, in our experiments, a strong rectification appears as we tune the thickness of the BTB layer to leave the tunneling regime. Change in the film morphology during the growth process could also play a role. ${ }^{37-39}$ 


\section{CONCLUSION}

In this article, we have shown the ability to obtain robust, ultrathin and large area molecular junctions including an electro-grafted molecular film. We also report tunneling through this organic layer when its thickness reaches few BTB monomers length. Its thickness can be tuned up to $27 \mathrm{~nm}$ thanks to our technique but, we believe it can be enlarge over a wider range by changing the limits of the scan window, the scan rate and the BTB concentration. This results are important for the development of future molecular based electronic devices.

\section{ACKNOWLEDGEME NTS}

We acknowledge P. Filloux, S. Suffit and C. Manquest for technical support within the cleanroom of the University Paris Diderot. This work is supported by LABEX SEAM. Commissariat général à l'investissement d'avenir (CGI) and ANR are acknowledged for their financial support to labex SEAM.

\section{REFERENCES}

${ }^{1}$ S. V Aradhya and L. Venkataraman, Nat. Nanotechnol. 8, 399 (2013).

${ }^{2}$ A. Batra, P. Darancet, Q. Chen, J.S. Meisner, J.R. Widawsky, J.B. Neaton, C. Nuckolls, and L. Venkataraman, Nano Lett. 13, 6233 (2013).

${ }^{3}$ K. Smaali, S. Karpe, P. Blanchard, D. Deresmes, S. Godey, A. Rochefort, J. Roncali, and D. Vuillaume, ACS Nano 4, 2411 (2010).

${ }^{4}$ M. Mannini, F. Pineider, P. Sainctavit, C. Danieli, E. Otero, C. Sciancalepore, A.M. Talarico, M.-A. Arrio, A. Cornia, D. Gatteschi, and R. Sessoli, Nat. Mater. 8, 194 (2009).

${ }^{5}$ C. Barraud, P. Seneor, R. Mattana, S. Fusil, K. Bouzehouane, C. Deranlot, P. Graziosi, L. Hueso, I. Bergenti, V. Dediu, F. Petroff, and A. Fert, Nat. Phys. 6, 615 (2010).

${ }^{6}$ V. Rabache, J. Chaste, P. Petit, M.L. Della Rocca, P. Martin, J.-C. Lacroix, R.L. McCreery, and P. Lafarge, J. Am. Chem. Soc. 135, 10218 (2013).

${ }^{7}$ M.M. Ling and Z. Bao, Chem. Mater. 16, 4824 (2004).

${ }^{8}$ D. Vuillaume, in Oxford Handb. Nanosci. Nanotechnology. Front. Adances (2008). 
${ }^{9}$ N.J. Tao, Nat. Nanotechnol. 1, 173 (2006).

${ }^{10}$ H. Haick and D. Cahen, Prog. Surf. Sci. 83, 217 (2008).

${ }^{11}$ H. Yan, A.J. Bergren, R. McCreery, M.L. Della Rocca, P. Martin, P. Lafarge, and J.-C. Lacroix, Proc. Natl. Acad. Sci. U. S. A. 110, 5326 (2013).

${ }^{12}$ S.Y. Sayed, J.A. Fereiro, H. Yan, R.L. McCreery, and A.J. Bergren, Proc. Natl. Acad. Sci. U. S. A. 109, 11498 (2012).

${ }^{13}$ M.P. Stewart, F. Maya, D. V Kosynkin, S.M. Dirk, J.J. Stapleton, C.L. McGuiness, D.L. Allara, and J.M. Tour, J. Am. Chem. Soc. 126, 370 (2004).

${ }^{14}$ J. Pinson and F. Podvorica, Chem. Soc. Rev. 34, 429 (2005).

${ }^{15}$ D. Bélanger and J. Pinson, Chem. Soc. Rev. 40, 3995 (2011).

${ }^{16}$ X. Lefèvre, O. Segut, P. Jégou, S. Palacin, and B. Jousselme, Chem. Sci. 3, 1662 (2012).

${ }^{17}$ A.P. Bonifas and R.L. McCreery, Nano Lett. 11, 4725 (2011).

${ }^{18}$ H. Yan, A.J. Bergren, and R.L. McCreery, J. Am. Chem. Soc. 133, 19168 (2011).

${ }^{19}$ A.J. Bergren, R.L. McCreery, S.R. Stoyanov, S. Gusarov, and A. Kovalenko, J. Phys. Chem. C 114, 15806 (2010).

${ }^{20}$ R.L. McCreery, H. Yan, and A.J. Bergren, Phys. Chem. Chem. Phys. 15, 1065 (2013).

${ }^{21}$ M. Delamar, R. Hitmi, J. Pinson, and J.M. Saveant, J. Am. Chem. Soc. 114, 5883 (1992).

${ }^{22}$ P. Martin, M.L. Della Rocca, A. Anthore, P. Lafarge, and J.-C. Lacroix, J. Am. Chem. Soc. 134, 154 (2012).

${ }^{23}$ C. Fave, Y. Leroux, G. Trippé, H. Randriamahazaka, V. Noel, and J.-C. Lacroix, J. Am. Chem. Soc. 129, 1890 (2007).

${ }^{24}$ C. Fave, V. Noel, J. Ghilane, G. Trippé-Allard, H. Randriamahazaka, and J.-C. Lacroix, J. Phys. Chem. C 112, 18638 (2008).

${ }^{25}$ D.R. Jayasundara, R.J. Cullen, and P.E. Colavita, Chem. Mater. 25, 1144 (2013).

${ }^{26}$ A. Laforgue, T. Addou, and D. Bélanger, Langmuir 21, 6855 (2005).

${ }^{27}$ T. Hines, I. Díez-Pérez, H. Nakamura, T. Shimazaki, Y. Asai, and N. Tao, J. Am. Chem. Soc. 135, 3319 (2013).

${ }^{28}$ S. Ho Choi, B. Kim, and C.D. Frisbie, Science 320, 1482 (2008).

${ }^{29}$ D.A. Rabson, B.J. Jönsson-Åkerman, A.H. Romero, R. Escudero, C. Leighton, S. Kim, and I.K. Schuller, J. Appl. Phys. 89, 2786 (2001).

${ }^{30}$ Z.-S. Zhang, J. Appl. Phys. 95, 557 (2004). 
${ }^{31}$ M.L. Perrin, C.J.O. Verzijl, C.A. Martin, A.J. Shaikh, R. Eelkema, J.H. van Esch, J.M. van Ruitenbeek, J.M. Thijssen, H.S.J. van der Zant, and D. Dulić, Nat. Nanotechnol. 8, 282 (2013).

${ }^{32}$ S.H. Choi, C. Risko, M.C.R. Delgado, B. Kim, J.-L. Brédas, and C.D. Frisbie, J. Am. Chem. Soc. 132, 4358 (2010).

${ }^{33}$ S.-C. Chang, Z. Li, C.N. Lau, B. Larade, and R.S. Williams, Appl. Phys. Lett. 83, 3198 (2003).

${ }^{34}$ N. Nerngchamnong, L. Yuan, D.-C. Qi, J. Li, D. Thompson, and C.A. Nijhuis, Nat. Nanotechnol. 8, $113(2013)$.

${ }^{35}$ G.L. Fisher, A. V. Walker, A.E. Hooper, T.B. Tighe, K.B. Bahnck, H.T. Skriba, M.D. Reinard, B.C. Haynie, R.L. Opila, N. Winograd, and D.L. Allara, J. Am. Chem. Soc. 124, 5528 (2002).

${ }^{36}$ H. Ishii, K. Sugiyama, E. Ito, and K. Seki, Adv. Mater. 11, 605 (1999).

${ }^{37}$ Y. Zilberman, U. Tisch, G. Shuster, W. Pisula, X. Feng, K. Müllen, and H. Haick, Adv. Mater. 22, 4317 (2010).

${ }^{38}$ N. Gozlan, U. Tisch, and H. Haick, J. Phys. Chem. C 112, 12988 (2008).

${ }^{39}$ D. Knipp, R.A. Street, A. Völkel, and J. Ho, J. Appl. Phys. 93, 347 (2003). 